\title{
Michel Houellebecq oder die Unterwerfung der Liebe nach der Liebe
}

Es wäre $\mathrm{zu}$ verlockend gewesen, unsere Vorlesung mit dem von Gabriel García Márquez wunderschön angerichteten Glücksgefühl der erfüllten Liebe unserer zwei alten Leutchen aus Die Liebe in den Zeiten der Cholera zu beenden; doch ganz so wohlig und gemütlich wollte ich Sie nicht in das Leben außerhalb der Vorlesung entlassen. Beschäftigen wir uns also mit einem letzten Text aus dem Jahre 2015, der die Frage nach der Liebe im Kontext politischer Lektüren und einer Überschreitung der Grenzen weg von der Demokratie hin zur Diktatur in seinen Mittelpunkt rückt und damit den Zusammenhang zwischen Liebe und Politik, der uns im Verlauf unserer Vorlesung immer wieder beschäftigt hat, intensiv erkundet.

Der Protagonist von Michel Houellebecqs Roman Soumission ist ein Akademiker, genauer ein Literaturwissenschaftler und Spezialist für französische Literatur des 19. Jahrhunderts. Er hat die große Liebe seines Lebens, den Verfasser der ,Bibel' der Décadence und des Ästhetizismus, in den Gegenstand seiner philologischen Dissertation verwandelt, die er an der Université Paris IV - Sorbonne unter dem Titel Joris-Karl Huysmans, ou la sortie du tunnel einreichte und höchst erfolgreich verteidigte. Huysmans ist für uns wahrlich kein Unbekannter, denn wir haben uns in unserer Vorlesung ausführlich mit ihm beschäftigt! Die große Liebe ist für ihn folglich eine Lektüre.

Literaturwissenschaft und Literatur bilden gleichsam Gläser, durch welche in dem nachfolgend $\mathrm{zu}$ besprechenden Roman eine außersprachliche Wirklichkeit betrachtet wird, die zumindest einer französischen Leserschaft durch die Einbeziehung vieler zeitgenössischer und historischer Namen sowie einer Vielzahl nachprüfbarer Details sehr vertraut ist. Die Mimesis einer denkbaren französischen Wirklichkeit des Jahres 2022 und die intratextuellen Bezüge zu einem Schriftsteller des Fin de siècle bilden so das Textgewebe, aus dem dieser Roman gefertigt ist.

Rolle und Funktion der Literatur werden im Verlauf dieses Werkes immer wieder diskutiert und auf die dargestellten Ereignisse bezogen. So heißt es bereits kurz nach dem incipit, in dem sich der Protagonist namens François seiner ,triste jeunesse “1 und seiner frühen Liebe zu Huysmans erinnert:

Allein die Literatur kann Ihnen erlauben, in Kontakt mit dem Geist eines Toten zu treten, auf eine direktere, vollständigere und tiefere Art, als dies selbst die Konversation mit einem

1 Houellebecq, Michel: Soumission. Roman. Paris: Flammarion 2015, S. 11.

Ә Open Access. () 2020 Ottmar Ette, publiziert von De Gruyter. (๕)BY-NC-ND Dieses Werk ist lizenziert unter der Creative Commons Attribution-NonCommercial-NoDerivatives 4.0 International Lizenz. 


\begin{abstract}
Freund ermöglichen würde - ebenso tief und ebenso dauerhaft wie eine Freundschaft, denn man liefert sich in einer Konversation niemals so vollständig aus, wie man es vor einem leeren Blatt Papier tut, wenn man sich an einen unbekannten Adressaten wendet. Wohlgemerkt, geht es um die Literatur, dann besitzen die Schönheit des Stils, die Musikalität der Sätze ihre Wichtigkeit; die Tiefe der Reflexion des Autors, die Originalität seiner Gedanken sind nicht zu verachten; aber ein Autor ist vor allem ein menschliches Wesen, das in seinen Büchern gegenwärtig ist, möge er nun sehr gut oder sehr schlecht schreiben, ist dabei definitiv nicht von Belang, das Wesentliche ist, dass er schreibt und tatsächlich in seinen Büchern gegenwärtig ist [...]. ${ }^{2}$
\end{abstract}

Wir können aus den Leseerfahrungen unserer Vorlesung unschwer feststellen, dass dieses Zitat aus Houellebecqs Roman Soumission sich direkt an Marcel Prousts Sur la lecture annähert, auch wenn im Schlussteil dann die Akzente anders gesetzt werden. Denn das Lob des Schreibens und die Abwertung der Konversation sind große Themen nicht bei Huysmans, sondern beim Autor von A la recherche du temps perdu.

Diese Passage führt zugleich sehr schön die Vorgehensweise des textexternen Autors Michel Houellebecq - 1958 auf La Réunion geboren, bei einer Großmutter und im Internat aufgewachsen, zweimal geschieden und als Erfolgsautor und enfant terrible der französischen Gegenwartsliteratur eher zurückgezogen lebend - vor Augen, der zunächst zwar seine Figur namens François über sein Verhältnis zu Huysmans und, in einem zweiten Schritt, zur Literatur im Allgemeinen reflektieren lässt, dann aber in seinem Diskurs Aussagen platziert, welche die klare Grenzziehung zwischen textinternen und textexternen Sprecherpositionen zu verwirren und zu vervielfachen suchen.

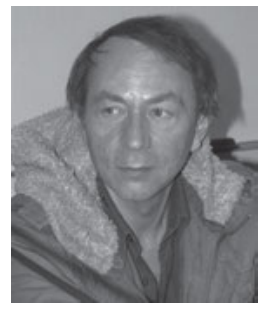

Abb. 120: Michel Houellebecq, eigentlich Michel Thomas (Saint-Pierre auf La Réunion, 1958), 2008.

Houellebecq geht es hier keineswegs darum, sich für den ,Inhalt‘ eines Textes zu Ungunsten der literarischen ,Form' auszusprechen, sondern gleichsam eine direkte Stimme des „menschlichen Wesens“ namens Autor innerhalb des Textes zu situieren und damit Möglichkeiten zu eröffnen, Aussagen sich selbst

2 Houellebecq, Michel: Soumission, S. 13f. 
als textexternem Autor zu attribuieren. Es geht damit um die Frage, welche und wessen Stimmen wir hören, wenn wir einen Text lesen. So handelt es sich um ein raffiniertes Verwirrspiel zwischen Romanfiguren und Autorfiguration, das der oft als Skandalautor Verschriene wiederholt sehr erfolgreich mit der Kritik gespielt hat. Denn sollen wir nicht Houellebecqs reale Stimme hören, wenn wir Worte und Sätze seiner Romanfiguren lesen, seien sie nun gut oder schlecht geschrieben? Die Art und Weise der Lektüre, die Funktionen und Modi des Lesens sind für die Romane dieses französischen Autors von zentraler Bedeutung.

Der Roman mit dem Titel Soumission, der am 7. Januar 2015 erschien, just dem Tag des mörderischen Terroranschlags auf die französische Satirezeitschrift Charlie Hebdo, ist sehr präzise durchkomponiert und gerade hinsichtlich seiner Möglichkeiten effizient, ein ständiges Verwirrspiel zwischen textinterner Figur und textexternem Autor in Gang zu setzen. Houellebecq provoziert ganz bewusst, als Rassist oder Frauenhasser, als Gegenaufklärer oder islamophober Anti-Intellektueller beschimpft zu werden. Erst das literarische Verfahren eines textuellen Quiproquo eröffnet alle Möglichkeiten einer polemischen, dem Autor oft vorgeworfenen provokativen Dimension seiner Texte, insofern nun dem Autor-Ich zugerechnet werden kann, was ,eigentlich' nur François denkt und schreibt, lebt und ausagiert. Michel Houellebecq, der von Rita Schober wiederholt in eine literaturgeschichtliche Reihe mit dem Naturalisten Emile Zola gestellt wurde, ${ }^{3}$ nutzt damit auf wirkungsvolle Weise raffinierte Textverfahren, um seine Figur François immer wieder als Sprachrohr vermeintlich eigener Ansichten zu nutzen.

Es kann wohl kaum ein Zweifel daran bestehen, dass Michel Houellebecq gegenwärtig „l'auteur le plus controversé de la littérature contemporaine française $^{\text {“4 }}$ ist und auf der Grundlage seiner „approche philosophique“ wie der „complexité scientifique“ seines Schreibens zugleich „une théorie de la vie“, 5 eine Theorie des Lebens zu entfalten versucht. Seien wir an dieser Stelle deutlich, ja kategorisch: Dies bedeutet weder auf Ebene einer solchen Lebenstheorie noch auf jener einer durch verschiedenste Äußerungen in Gang gesetzten Polemik, dass der reale Autor in seinen Texten direkt zu uns spräche und uns seine Ansichten unmittelbar kundtäte!

3 Vgl. das Kapitel „Renouveau du réalisme?“ in Schober, Rita: Auf dem Prüfstand. Zola - Houellebecq - Klemperer. Berlin: Walter Frey 2003, S. 195-207.

4 Diop, Ibou Coulibaly: Mondialisation et monde des théories dans l'œuvre de Michel Houellebecq. Mit einer ausführlichen deutschsprachigen Zusammenfassung. Berlin: Verlag Frank \& Timme 2018, S. 7.

5 Ebda. Diese Theorie des Lebens selbst erfolge geradezu unwillentlich (S. 155). 


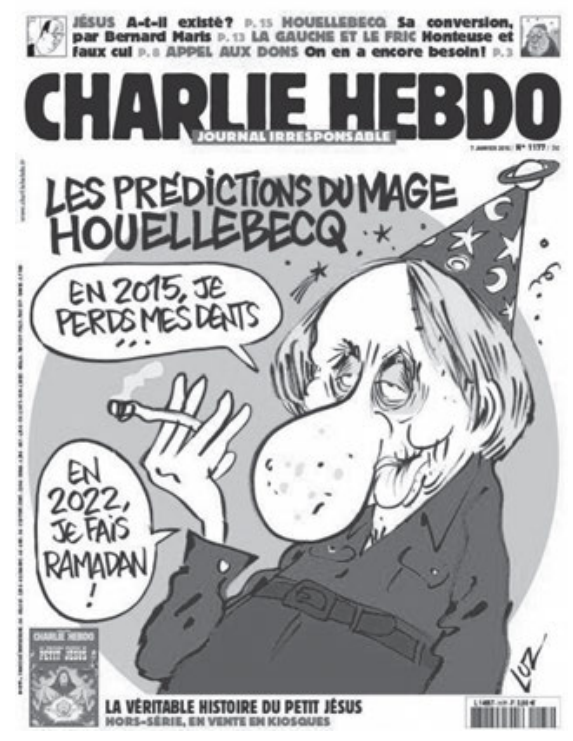

Abb. 121: Titelseite der Zeitschrift Charlie Hebdo, Ausgabe vom 7. Januar 2015.

Wir sollten uns vielmehr gerade mit Blick auf den in unserer Vorlesung erarbeiteten Lektürebegriff davor hüten, die Grenze zwischen textinternen und textexternen Erzählinstanzen niederzureißen! Denn bei François haben wir es mit einer von Houellebecq klug und überlegt geschaffenen Figur zu tun, die keineswegs für den textexternen Autor einsteht oder dessen Gewährsmann wäre. Autor und Autorschaft, Autorität und Autorisierung fallen bei diesem Erzählmodell nicht in eins. So müssen wir zur Kenntnis nehmen, dass die Wirkungsmacht sowie das Skandalisierungspotential des Schreibmodells Michel Houellebecqs in diesem wie auch anderen Romanen des französischen Schriftstellers von einer gezielten Vervielfachung und Verwischung der Grenzen ausgehen. Soumission ist kein gesellschaftspolitisches Traktat, basiert sehr wohl aber auf politischen und gesellschaftlichen, kulturellen und demographischen Analysen, denen man eine gewisse Stringenz nicht absprechen kann.

Gleichzeitig sollten wir nicht der Versuchung erliegen, Leben und Literatur strikt durch eine feste Grenze voneinander zu trennen! Wir haben in unserer Vorlesung von Beginn an und ausführlich die komplexen Zusammenhänge zwischen Leben, Lieben und Lesen gesehen. Denn Literatur und Leben sind aufs Engste miteinander verbunden, ohne selbstverständlich miteinander deckungsgleich zu sein. Gerade in den neueren Interviews - etwa in einem kurz nach dem Erscheinen von Soumission und den Anschlägen auf Charlie Hebdo mit Iris Radisch für die ZEIT geführten - hat Houellebecq selbst immer wieder ironisch darauf verwiesen, dass es zwischen ihm und seinen Figuren durchaus Unterschiede in den 
Einschätzungen und Überzeugungen gebe. ${ }^{6}$ Mit seinem Leben aber haben all diese Figuren sehr viel zu tun; Literatur und Leben sind auf den unterschiedlichsten Ebenen friktional miteinander vermittelt und verknüpft.

Dies wird auch auf der Textebene selbst eindrücklich in Szene gesetzt: Die enorme Bedeutung, welche Leben und Werke des Joris-Karl Huysmans für die literarische Figur François besitzen, bildet dank ständiger Einblendungen von Textfragmenten Huysmans' das narrative wie diskursive Rückgrat von Houellebecqs Roman. Der décadent steht freilich nicht für die Komfortzone des Literaturwissenschaftlers; Huysmans bleibt für diesen nicht nur intimer Freund, sondern stetiger Herausforderer in einer Gegenwart ohne Sinn, Sinnhaftigkeit und Sinnlichkeit. Es ist gerade diese Sinnsuche seines Lesevorbilds wie intertextuellen Modells, die in der Figur François in ihrer ganzen Abgründigkeit von Michel Houellebecq zutage gefördert wird.

Durch die Literatur wird zugleich die Grenze zwischen Leben und Tod durchlässig, geht es in ihr doch um den „,contact avec l'esprit d'un mort“, ${ }^{7}$ den Kontakt mit dem Geist eines Toten. Insofern beinhaltet und transportiert die Literatur nicht nur ein Lebenswissen und Überlebenswissen, sondern zugleich ein Weiterlebenswissen, ${ }^{8}$ das die vermeintlich so scharfen Grenzen zwischen Leben und Tod unterläuft. Die Literatur verfügt hier über eine sehr spezifische Form des Wissens, die durch Lektüre angeeignet werden kann; die Grenzen des Lebens sind von den Grenzen des Lesens nicht unabhängig.

Huysmans wird dadurch keineswegs $\mathrm{zu}$ einem Wiedergänger oder $\mathrm{zu}$ einem Huysmans redivivus; vielmehr werden Leben und Werke des Autors von A rebours für François zu einem Interpretament, in dessen Koordinatensystem sich das Handeln der Romanfigur immer wieder verortet und befragt. In Huysmans' eigener Deutung von des Esseintes spiegelt sich geschickt die Liebe von François zum Verfasser von A rebours. Mit Blick auf andere Romane des französischen Autors wird diese Konfiguration zum „Laboratorium eines Lebens“9 und dieses literarische Laboratorium seinerseits zu einem Modell der Welt, zu einem WeltFraktal, ${ }^{10}$ in dem uns in einem literarisch modellierten Mikrokosmos durch Prozesse wechselseitig projizierter Selbstähnlichkeit grundlegende Einsichten

6 Houellebecq, Michel: Der Tod ist nicht auszuhalten. Interview von Iris Radisch. In: Die Zeit (Hamburg) (23.1.2015).

7 Houellebecq, Michel: Soumission, S. 13.

8 Vgl. Ette, Ottmar: Welterleben / Weiterleben. On Vectopia in Georg Forster, Alexander von Humboldt, and Adelbert von Chamisso. In: Daphnis (Amsterdam) 45 (2017), S. 343-388.

9 Diop, Ibou Coulibaly: Mondialisation, S. 14.

10 Vgl. Ette, Ottmar: WeltFraktale. Wege durch die Literaturen der Welt. Stuttgart: J.B. Metzler Verlag 2017. 
in einen textexternen Makrokosmos ästhetisch zu Bewusstsein gebracht werden können. Huysmans ist durch die wiederholten Lektüren und literaturwissenschaftlichen Recherchen von François in der Gegenwart präsent und eröffnet Möglichkeiten, denkbare Wege in die Zukunft abzustecken. Zugleich aber wird seine Sinnsuche nach christlicher Transzendenz in ihr islamisches und mehr noch islamistisches Zerrbild verkehrt.

Der Literaturwissenschaftler präsentiert sich uns von Beginn an als ein zwar mit den Regeln im akademischen Feld wohlvertrauter Spezialist für die französische Literatur des 19. Jahrhunderts, ist ansonsten aber von einer völligen Sinnleere erfasst, die mit seiner eigenen Tätigkeit als Universitätslehrer beginnt; denn literaturwissenschaftliche Studien führten, das wisse man ja, „zu mehr oder minder nichts“!11 Es handele sich um ein System, das nur der eigenen Reproduktion von Spezialisten diene und ansonsten über 95 Prozent Abfall („déchet“) ${ }^{12}$ produziere, wenn man einmal außer Acht lasse, dass gebildete Studentinnen auf dem Arbeitsmarkt größere Chancen hätten, als Verkäuferinnen angestellt zu werden. Das Lachen des französischen Autors ist gewiss durch die Lektüre hindurch noch deutlich zu hören ...

Auch in anderer Hinsicht funktioniert die Universität als geschlossenes System: François selbst wählt seine Liebespartnerinnen jeweils semesterweise aus der Studentinnenpopulation aus, wobei er zeitweise polygame, später eher monogame Frauenbeziehungen bevorzugt. Sehr rasch zeigt sich François' Misogynie, eine grundlegende Frauenfeindlichkeit, die letztere lediglich als rasch dem physischen Verfall preisgegebene Lustobjekte betrachtet. Gleichaltrige Kolleginnen kommen da für den Mittvierziger schon lange nicht mehr wirklich in Frage. Dies wird an einigen Szenen durchgespielt, die nicht nur - wie oft bei Houellebecq - sexually explicit sind, sondern brutal aus patriarchalischer Sicht Frauen in ihrem körperlichen Verfall vorführen; ein Element, das im weiteren Verlauf des Romans innerhalb polygamer Beziehungen im Schutze des Islam resemantisiert werden wird. Abendländische Misogynie und morgenländische Frauenausbeutung werden im Frankreich des Jahres 2022 schon bald eine unheilige Allianz eingehen. Huysmans' ästhetisch bedingte Abkehr von der Liebe als Romansujet gerät bei François offenkundig zu einer phallogozentrischen Sichtweise heterosexueller Liebe, welche sich als Liebe nach der Liebe entpuppt.

Da weite Teile von Soumission im universitären Milieu spielen, lassen sich in diesem Text zweifellos Elemente eines Campus-Romans ausmachen: eine

11 Houellebecq, Michel: Soumission, S. 17.

12 Ebda. 
kleine Welt mit ihren Regeln, Hierarchien und oft erstaunlichen Gepflogenheiten. Zugleich ist Der Campus als Small World - um die Titel der Romane von Dietrich Schwanitz und David Lodge miteinander zu kombinieren - der Mikrokosmos einer größeren Welt, in der wichtige politische Gewichtverschiebungen, ja seismische Erschütterungen vor sich gehen. In dem Maße, in dem sich das politische Leben Frankreichs in den Roman drängt, wird aus dem Campus-Roman ein historischer Roman, in welchem sich historisch beglaubigte Figuren französischer Politiker mit ihren leicht identifizierbaren Namen um eine erfundene Zentralfigur gruppieren, die in Gestalt von François gewiss keine Identifikationsfigur darstellt.

Denn aus seiner Perspektivik, die von (mehr oder minder aufgeklärtem) Rassismus, Kolonialismus, Antisemitismus und Misogynie gekennzeichnet ist, wird eine Welt in Umbrüchen skizziert, in welcher nach langen Jahren einer als langweilig beschriebenen Machtteilung zwischen eher linken und eher konservativen Regierungen nun, im Jahre 2022, im Vorfeld der Präsidentschaftswahlen in Frankreich ebenso die rechtsradikalen und identitären wie die islamistischen und fundamentalistischen Bewegungen auf dem Vormarsch sind und letztlich die bisherigen demokratischen Parteien an den Rand drängen. Der historische Roman mutiert hier zum Zukunftsroman und begibt sich in ein futur proche von hoher Aktualität und keineswegs ausschließbarer Wahrscheinlichkeit: Houellebecq hat eine verteufelt gute Fiktion erfunden, in der wir die Faktizität der Dinge neu zu beleuchten lernen! Insofern ergeben sich Züge einer Dystopie, droht die bisher zwischen zwei politischen Lagern fein säuberlich aufgeteilte Welt doch nun in gewalttätige Auseinandersetzungen, einen blutigen Bürgerkrieg und einen letztlich demokratiefeindlichen, diktatorischen Regimetypus abzugleiten. Denn wir sehen Frankreich nicht an der Schwelle zum Bürgerkrieg: Der Bürgerkrieg hat vielmehr im Vorfeld der Présidentielles bereits begonnen.

Der Einbruch der politischen Veränderungen in die heile akademische Welt erfolgt bereits zu Beginn des zweiten Teiles des Romans, als während einer netten Gartenparty der Spezialisten für die französische Literatur des 19. Jahrhunderts plötzlich und in unmittelbarer Nähe mitten in Paris längere Schusswechsel sowie Detonationen hörbar werden. Man ist beunruhigt, aber man spricht noch etwas über Huysmans oder Mallarmé, verabschiedet sich, hofft, dass die eigene Wohnung nicht betroffen sein möge, enthält sich ansonsten aber jeglicher Stellungnahme. Niemand scheint sich hier für die Aufrechterhaltung eines demokratischen Systems engagieren zu wollen. Das akademische System erscheint als eine kleine, in sich geschlossene und nur auf sich selbst gerichtete Welt, in der für Politik kein Platz ist. Dies wird im Roman Folgen haben ...

Überhaupt scheinen viele die Lage nicht für gefährlich zu halten - und Parallelen zum zeitgenössischen Frankreich sind auch hier nicht ausgeschlossen. 
Staatliche Sicherheitsbeamte mit umgehängten Maschinenpistolen schlendern angeregt plaudernd und mit heiterer Mine vorbei; François fällt es schwer zu begreifen, warum sie sich um nichts zu kümmern scheinen, so als wäre nichts geschehen: „ils font absolument comme si de rien n'était.“13 Längst hat eine grundlegende Normalisierung stattgefunden, in welcher der Ausnahmezustand ${ }^{14}$ den Blick auf eine Welt jenseits der Demokratie freigibt. Und rasch zeigt sich, dass einer der Kollegen mit dem sprechenden Namen Lempereur - so wie bei Flaubert der Händler Lheureux - über beste Verbindungen zur identitären Bewegung verfügt, ja möglicherweise seit längerer Zeit schon als einer ihrer Vordenker fungiert. Nichts in der akademischen Welt regt sich, um für den Erhalt der Demokratie einzutreten oder gar zu kämpfen; sie ist kein Hort des Widerstands in diesem zeitkritischen Roman voll beißender Ironie. Man ist versucht, die Frage zu stellen, ob sich denn in unserer akademischen Welt Widerstand regen würde, und schaudert vor der Antwort zurück ... Nein, die Universität war - allen Göttinger Sieben zum Trotz - noch nie ein Hort des Widerstands gegen autoritäre Regime!

So zeigt sich in dieser von Houellebecq hintergründig arrangierten Szenerie bereits modellhaft, dass eine Vielzahl von Grenzen in Richtung Diktatur überschritten werden, ohne dass von der Welt der Akademiker auch nur die geringste Aktivität zugunsten demokratischer Prozesse und Verfahren ausginge. Man fürchtet nur um die eigene Sicherheit, um das eigene Hab und Gut, um die eigene Wohnung oder das eigene Auto. François bleibt ruhig und wie stets lakonisch; er begleitet Lempereur und nimmt durchaus mit Interesse zur Kenntnis, in welchem Denksystem und Diskursuniversum sich dieser Kollege bewegt, der von seiner Machstellung innerhalb rechtsradikaler Bewegungen offenkundig auch finanziell stark profitiert und an der Vorbereitung eines Bürgerkrieges maßgeblich beteiligt ist. Deutlich lesbar wird in Lempereurs luxuriös ausgestatteter Wohnung der Titel einer Schrift zur Vorbereitung eines Bürgerkrieges: „PREPAREZ LA GUERRE CIVILE“. ${ }^{15}$

Das eher sanfte, kollegiale Eintauchen in ein rechtsradikales Diskursuniversum führt vor, wie sehr sich die Logosphäre im Vorfeld der anstehenden Präsidentschaftswahlen in Frankreich zwar unmerklich, aber nachhaltig verändert hat. Ebenso im rechtsradikalen Spektrum und bei Marine Le Pens Front National wie in den unterschiedlichen islamistischen Bewegungen ist 2022 der Antisemitismus wieder gesellschaftsfähig; und dies nicht nur auf der politischen Ebene, sondern auch im Alltagsleben. François wird davon direkt betroffen, denn

13 Ebda., S. 69.

14 Zur Normalität des Ausnahmezustands vgl. Agamben, Giorgio: Stato di eccezione. Homo sacer, II, 1. Torino: Bollati Boringhieri 2003.

15 Houellebecq, Michel: Soumission, S. 74. 
seine derzeitige Sexpartnerin Myriam, deren junge und erregende Körper- und Geschlechtsteile er liebt und in unterschiedlichen Stellungen der Unterwerfung für sich lustgewinnend nutzt, ${ }^{16}$ wird wie ihre ganze Familie in einer immer offener antisemitischen Atmosphäre zum Verlassen Frankreichs und zur Flucht nach Israel gezwungen. François bedauert dies zwar, denn keine andere junge Studentin gestaltet seine Liebesnächte und Wochenenden so angenehm und aufregend wie Myriam, der er viel Sympathie entgegenbringt. Aber als ihrer Familie schließlich keine andere Wahl mehr bleibt, als nach Israel zu emigrieren, gewöhnt er sich rasch an den Gedanken. Er akzeptiert die neue Situation in einem zutiefst antisemitischen Frankreich ohne Murren; denn letztlich hat er keineswegs seine Liebe verloren, sondern nur ein erotisch erregendes Sexual- und Lustobjekt, das er jederzeit wieder ersetzen kann. Wir haben es offenkundig mit der Liebe nach der Liebe zu tun! Wie schon bei Huysmans hat die große, die das ganze Wesen des Menschen erfassende Liebe längst abgedankt; Myriam ist für François bestenfalls eine neue Salomé.

Damit ist zugleich eine weitere der vielen Grenzen überschritten; die Logosphäre verändert sich immer rascher hin zu militanten Ausdrucksformen von Exklusion, wobei die Konfrontationen nicht nur verbal, sondern zunehmend gewalttätig ausgetragen werden. Gewalt rückt immer mehr ins Zentrum des Romans. Auf der Ebene sexueller Befriedigung hält François sich im Übrigen schadlos ... Wie andere männliche Protagonisten Houellebecqs findet auch hier, wie Rita Schober lange vor dem Erscheinen von Soumission formulierte, eine „Unterwerfung der Geschlechter in der Liebeskonkurrenz unter die Marktgesetzlichkeit“"17 statt. Die kapitalistische Warenordnung, deren aufkommen wir in Gertrudis Gómez de Avellanedas Roman Sab hatten beobachten können und etwa bei Flauberts Madame Bovary in ihrem weiteren Fortschritt begleitet hatten, ist in der von Michel Houellebecq skizzierten Gesellschaft omnipräsent und dominant geworden.

Der Roman setzt diesen zu Beginn fast unmerklichen Prozess eines immer schnelleren Überschreitens von Grenzen in Richtung einer in ihren Konturen noch offenen autoritären Herrschaft meisterhaft in Szene. Der anfängliche Campus-Roman ist zu einem historischen und dieser zu einem politischen Roman geworden, ohne dass die Verbindungen zwischen diesen einzelnen Subgattungen gekappt würden. Der Campus ist eine ganze Welt, so wie François schon mit seinem Namen für die Welt der Franzosen einsteht. In vielen kleinen, fast unmerklichen Schritten zeigt sich, wie eine Demokratie in eine Diktatur

16 Ebda., S. 108-113.

17 Schober, Rita: Auf dem Prüfstand, S. 260. 
beziehungsweise ein autoritäres Regime übersetzt werden kann und abgleitet. Dazu bedarf es nur weniger grundlegender Veränderungen, wohl aber einer Querung zahlreicher Grenzen, die freilich ohne größeren Widerstand überwindbar sind.

Längst ist in der Konvivenz verschiedener politischer, kultureller und religiöser Gemeinschaften ein Zusammenlebenswissen geschwunden, ja fast schon verschwunden, das von unterschiedlichen Seiten absichtsvoll zersetzt wird, um ein friedliches Zusammenleben populistisch aufkündigen zu können. Die demokratischen Strukturen funktionieren noch, die Präsidentschaftswahlen werden abgehalten und trotz aller Probleme auch in der zweiten Runde durchgeführt. Die traditionellen demokratischen Parteien Frankreichs verschwinden dabei jedoch; eine völlig neue Parteienlandschaft beginnt sich zu konstituieren. Der Roman macht es schwer, ja bewusst unmöglich, die genaue Grenze zu bestimmen, an welcher eine Demokratie sich in ihr Gegenteil verkehrt. Es gibt diese eindeutige Grenze nicht, es gibt nur eine Vielzahl von Grenzen.

An die Bilder vom Zusammenbruch einer alten Ordnung, die gerade den Beginn des dritten Teiles durchziehen, setzt sich immer mehr die Silhouette einer neuen Ordnung, welche dank der gut vorbereiteten Strukturen der islamischen Bruderschaften nur einen einzigen wirklichen Nutznießer kennt. Spätestens mit dem Wahlsieg über Marine Le Pen in der zweiten Runde der Präsidentschaftswahlen sehen wir unter der geschickten Führung des neuen Präsidenten Ben Abbes eine autoritäre Führung islamischen bis islamistischen Zuschnitts heraufziehen, die es versteht, die von ihr angestrebten politischen und gesellschaftlichen Umwälzungen mit dem Verweis auf die Herstellung rascher politischer Stabilität zu versehen. Dieses Versprechen lockt, wie die Aussicht auf stabile Verhältnisse immer schon ein Trumpf autoritärer Herrschaftsordnungen war. Allein auf diese Weise scheint ein Bürgerkrieg in Frankreich noch vermeidbar zu sein.

Nach dem Wahlsieg von Ben Abbes werden binnen kürzester Zeit die Sorbonne und andere staatliche Universitäten gleichgeschaltet, die Frauen ihrer Dozentenstellung beraubt und islamistische Universitätsstrukturen geschaffen, ohne dass sich innerhalb wie außerhalb der universitären Mauern auch nur im geringsten Widerstand bemerkbar machen würde. Die Universität erscheint erneut als Fraktal der gesamten französischen Gesellschaft, doch das universitäre Milieu ist erfreut: Denn die Einführung der Polygamie knüpft nahtlos an die Träume vieler Mitglieder des Professorats und Patriarchats an, wobei den Professoren nach Bedarf Studentinnen zur Verfügung gestellt werden, die sich aufopferungsvoll ihren Studien wie ihren neuen Aufgaben jeweils mit und ohne Burka zuwenden. Die Unfähigkeit zur Liebe bei Männern wie bei Frauen, wie wir sie in den vorgängigen Romanen Michel Houellebecqs überdeutlich skizziert finden, 
wird hier als Suche nach Liebe gleichsam religiös instrumentalisiert und in eine scheinbar höhere Sphäre gehoben. Staatlich beglaubigte Kupplerinnen kümmern sich erfolgreich um die Eheanbahnungen im Zeichen des Islam; Liebe wird in dieser Fiktion mit dem Schleier der Transzendenz verbrämt.

Auch auf anderen Ebenen gewinnt das Pariser Patriarchat in Soumission der nunmehr religiös begründeten Geschlechterhierarchie zahlreiche positive Seiten ab: Die ehemaligen Kolleginnen sind längst zu herausragenden Köchinnen geworden, die sich zur Freude ihrer Männer ganz auf ihre neue alte Aufgabe am Kochtopf konzentrieren. Nicht einmal Gewalt muss angewendet werden; Misogynie und Phallogozentrismus der westlich geprägten europäischen Gesellschaften gehen hier geradezu sanft und widerspruchsfrei in eine kulturell und religiös fundierte Männerherrschaft ein, die sich den neuen Gesetzen gerne beugt, weil sie sich dadurch die Frauen zu unterwerfen vermag. Kein Zweifel: Wir haben es mit einer Dystopie zu tun! Aber die Arbeitslosigkeit in Frankreich sinkt, denn die Frauen verschwinden zunehmend vom Arbeitsmarkt; ihr Platz ist fortan (wieder) zuhause. Eine neue, nicht länger vom Bürgerkrieg bedrohte stabile Gesellschaftsordnung autoritären Zuschnitts ist noch im Wahljahr 2022 an die Stelle der längst überkommenen Gesellschaften westlich-demokratischer Tradition getreten. Die Demokratie hat sich selbst abgewählt, hat sich selbst abgeschafft.

Nicht nur innerhalb der Universitätslandschaft, sondern auch außerhalb des akademischen Feldes haben sich zunächst fast unmerklich Leben und Zusammenleben der Menschen verändert. François hat anfänglich Mühe zu erkennen, woran dieser grundlegende Wandel etwa auf der Straße, im Alltagsleben, beim Shopping in den großen Einkaufszentren, festgemacht werden kann und sichtbar wird. Der Franzose flaniert zu Beginn von Teil IV, unmittelbar nach der Machtübernahme der Bruderschaft, durch die Straßen von Paris und registriert aufmerksam, dass es weniger die Geschäfte selbst sind als „le public en lui-même qui avait, subtilement, changé“, ${ }^{18}$ das Publikum selbst hatte sich augenscheinlich verwandelt! Die Veränderungen zeichnen sich erst langsam ab und werden nicht sofort bemerkbar:

Und die weibliche Kleidung hatte sich verändert, ich fühlte es sofort, ohne dass es mir freilich gelungen wäre, diese Verwandlung zu analysieren; die Zahl islamischer Schleier hatte sich kaum vergrößert, das war es nicht, und ich brauchte fast eine Stunde des Herumspazierens, bis ich auf einen Schlag erfasste, was sich verändert hatte: Alle Frauen steckten jetzt in Hosen. Die Enthüllung weiblicher Oberschenkel, die mentale Projektion des weiblichen Geschlechtsteiles in den Schritt, ein Prozess, dessen erregende Macht unmittelbar proportional von der Länge der entblößten Beine abhängt: All dies war bei mir so unwillentlich und maschinenhaft, so genetisch in gewisser Weise, dass es mir nicht sofort bewusst

18 Houellebecq, Michel: Soumission, S. 185. 
wurde, aber das Faktum war da, alle Kleider und Röcke waren verschwunden. Zugleich hatte sich eine neue Bekleidung verbreitet, eine Art langer Baumwollbluse, die bis zur Mitte der Oberschenkel reichte und jegliches objektives Interesse an den körperbetonten Hosen raubte, die gewisse Frauen eventuell noch hätten tragen können; was die Shorts betraf, so war es klar, dass es darum schon längst nicht mehr ging. Selbst die Betrachtung weiblicher Pos war als minimaler träumerischer Trost fortan unmöglich geworden. ${ }^{19}$

Im Reich der Zeichen ${ }^{20}$ non-verbaler wie verbaler Art reagiert das System der Mode $^{21}$ wie ein kollektives Frühwarnsystem, das die grundlegenden Veränderungen durch sanfte Anpassungsmodi manifestiert. Seismographisch genau übersetzen sich die gesellschaftlichen und politischen Veränderungen in Verrückungen der Grenzen von Sichtbarkeit und Sichtbarmachung am weiblichen Körper, der als Spielfläche männlicher Projektionen neu vermessen, kartographiert und semantisiert wird. Mit Liebe hat dies alles nichts mehr zu tun; hier geht es nur noch um Körper, Sexualität, Macht und Gewalt!

Dabei sind es gerade nicht die ostentativen und semantisch klar definierten weiblichen Kleidungsstücke wie Schleier oder Burka, sondern die Vervielfachungen von vestimentären Grenzziehungen, welche die politischen Transformationen in alltägliche Grenzen übersetzen, an denen die Bewegungen zwischen einer Demokratie westlichen Typs und einem autoritären Regime nach islamistischem Vorbild in die Sprache weiblicher Kleidung übertragen werden. Es sind folglich keineswegs allein diskursive und verbale Übersetzungen, sondern Zeichensysteme unterschiedlichster Art, innerhalb derer die Translationsprozesse ablaufen. Das gesamte System kultureller Ausdrucksformen wird neu kodiert; eine neue Politik, aber auch eine neue Poetik und Polemik der Grenze ist entstanden, anschaulich vermessen am Körper der Frauen, die einmal mehr entrechtet und zu Objekten gemacht werden.

Die Allgegenwart von Joris-Karl Huysmans ist auch in diesen Teilen des Romans ungebrochen, in denen es um die radikale Umgestaltung einer demokratischen Gesellschaft geht sowie um den Umbau der Europäischen Union in ein künftiges Europa nach dem Vorbild des Römischen Reiches - freilich unter der Vorherrschaft des Islam. Der Werteverfall war im literarischen Kosmos wie in der Lebensgeschichte des finisekulären Literaten gerade in der übersteigerten Sinnsuche deutlich geworden, und eben deshalb wird Huysmans für Houellebecq zum intertextuellen Referenzpartner. François selbst lernt als ständiger Leser des Verfassers der ,Bibel` aller Décadents buchstäblich immer neue Seiten am von

19 Ebda., S. $185 f$.

20 Barthes, Roland: L’Empire des Signes. Paris - Genève: Flammarion - Skira 1970.

21 Barthes, Roland: Système de la Mode. Paris: Seuil 1967. 
ihm verehrten französischen Autor kennen; er ist ein aufmerksamer, philologisch genauer Leser. Huysmans' Verhältnis zum Christentum hat Folgen für François' Beziehung zum Islam.

Dabei wird zunehmend deutlich, dass Huysmans nicht nur einer Reihe sexistischer Einstellungen von François Pate stand, sondern auch in seiner ferventen Glaubenssuche Strukturen weitergab, die es dem hochspezialisierten Literaturwissenschaftler später ermöglichen werden, sich der religiösen Herrschaft über die französische, wie auch perspektivisch über alle europäischen Gesellschaften freudig zu unterwerfen. Ganz so, wie es dem deutschen Literaturwissenschaftler Hans Robert Jauss gelang, sich allen Veränderungen anzupassen, die sich innerhalb seines Zeithorizonts ereigneten - von der nationalsozialistischen Diktatur über den Kampf gegen den Bolschewismus bis hin zur bundesrepublikanischen Demokratie und ihren Reformbewegungen -, gelingt es auch dem Literaturwissenschaftler François, seine Lektüren den jeweiligen Umständen anzuschmiegen. ${ }^{22}$

Daher überrascht es letztlich auch nicht, dass er sich nach einigem Zögern und opportunistischem Abwarten den neuen, islamistischen Spielregeln an der Sorbonne unterwirft und noch ein letztes Mal einen längeren Text über Huysmans verfasst, um den er gebeten wurde und um eine neue systemkonforme Lesart des Autors von En route vorzulegen, mit dessen als Motto vorangesetztem Zitat der Roman beginnt. Soumission stellt hier als Campus-Roman der Literaturwissenschaft von einer politisch-literarischen Warte aus ein wenig schmeichelhaftes Zeugnis aus, das im Übrigen auch Huysmans’ Kanonisierung zum Klassiker des Ästhetizismus und der Décadence nicht unbeschädigt lässt; denn die Politik der Unterwerfung beinhaltet stets nicht nur eine Polemik, sondern auch eine Poetik der Unterwerfung - und umgekehrt.

Der Ausklang des vierten und der Beginn des fünften und letzten Teiles entfalten diese Relation auf bohrende, insistierende Weise. Dies erfolgt vor dem Hintergrund einer politischen Entwicklung, in der die Vertreter der Identitären wie des Front National, die doch einst gegen die Islamisten kämpften und einen Bürgerkrieg gegen letztere, aber auch gegen die demokratisch verfassten Institutionen anzettelten, nun das Lager gewechselt haben und sich der neuen Sammlungsbewegung unter Ben Abbes und dessen islamischer Führung anschließen. Der neue französische Präsident befleißigt sich einer taktisch klugen und ausgewogenen Sprache, mit deren Hilfe für die ehemalige demokratische Mehrheitsgesellschaft

22 Vgl. hierzu Ette, Ottmar: Der Fall Jauss. Wege des Verstehens in eine Zukunft der Philologie. Berlin: Kulturverlag Kadmos 2016; vgl. hierzu auch die Rezension der französischen Ausgabe dieses Bandes durch Seurat, Alexandre: La philologie dévoyée par le non-dit: Jauss face à son passé SS. In: Acta fabula XXI, 1 (janvier 2020), 10 p. < http:/ /www.fabula.org/revue/document12572. php?fbclid=lwAR29ai_PMtaqim8KxYgihnvsFRbMRk3M2dOMAnRn8hs3Lcbmy9FXPK6Hes> . 
viele Brücken gebaut werden können, um von einem demokratischen zu einem nicht-demokratischen Gesellschaftssystem überzusetzen. Es geht alles ganz leicht und einfach ...

Die explizit vom britischen Kulturphilosophen Arnold J. Toynbee ${ }^{23}$ geborgte Grundthese des Romans, der zufolge Kulturen nicht durch Angriffe von außen, sondern durch ihre Zersetzung im Inneren zugrunde gehen, erfasst zweifellos die Rolle von Literaten wie Huysmans ebenso wie jene von Akademikern, die sich wie François nahezu bruchlos und ohne größere Schwierigkeiten nicht nur veränderten Universitäts-, sondern auch Gesellschaftssystemen anzupassen vermögen. Problemlos kann die Sorbonne von einem Jahre zuvor bereits zum Islam konvertierten Rektor Rediger, in dessen Figur sich die unterschiedlichen antidemokratischen Kräfte bündeln und zugleich mit einem hohen Bildungsstand verbinden, übernommen und in eine gegenüber dem neuen Staatswesen dienende Funktion überführt werden. Die Gleichschaltung der Universität, wie sie hinsichtlich der Universität Freiburg ebenfalls von einem hochgebildeten Rektor namens Martin Heidegger durchgeführt wurde, orientiert sich dabei zum einen an der von ihm gewonnenen Einsicht, dass Europa längst schon geistigen Selbstmord begangen habe, ${ }^{24}$ sowie an der Überzeugung, das höchste Glück des Menschen bestehe in seiner Unterwerfung; womit hier ebenso die sexuelle Unterwerfung der Frau unter die Macht des Patriarchats wie die Unterwerfung der Menschheit unter die Gebote einer islamistisch konsekrierten Diktatur verstanden wird. Unterwerfung wird an dieser Stelle zu einem transzendenten Begriff, durch welchen die Selbstaufgabe des Individuums normalisiert und religiös gerechtfertigt wird.

Vor diesem Hintergrund erstaunt es nicht, dass auch François - im Übrigen unter der populärphilosophischen Anleitung eines kleinen Büchleins von Rektor Rediger - zum Islam konvertiert und mit diesem Schritt ein zweites Leben beginnt, „la chance d'une deuxième vie, sans grand rapport avec la première“. ${ }^{25}$ Der Schlusssatz des Romans - ,Je n’aurais rien à regretter.“ (Ich werde nichts zu bedauern haben.) ${ }^{26}$ - steht für den vollzogenen Transformations-, Konversionsund Übersetzungsprozess von Demokratie in Diktatur und von einem westlichen Geschlechtermodell in eine islamistische Polygamie, in der alle Liebesbeziehungen gemäß Houellebecqs Fiktion neu definiert werden. Doch dieses westliche Modell hatte lange zuvor schon abgewirtschaftet und sich selbst, so will es der Roman wissen, ad absurdum geführt.

23 Houellebecq, Michel: Soumission, S. 268.

24 Ebda. S. 269.

25 Ebda., S. 315.

26 Ebda. 
Mit Liebe hat all dies nichts mehr zu tun; oder genauer: Wieder einmal zeigt uns die Literatur, wie eng verklammert Liebesbeziehungen mit politischen Beziehungen sind und in welch tiefgreifendem Maße Lesen, Leben und Lieben miteinander verkettet sind! Wenn in diesem Epilog die Liebe nach der Liebe zunehmend in den Mittelpunkt unserer Vorlesung gerückt wurde, so heißt dies nicht, dass damit das Spiel der Liebe an ein Ende gekommen wäre. Dies wird im Übrigen auch niemals der Fall sein, ist die revolutionäre Kraft der Liebe doch ein Faktor, der niemals aus unseren Gesellschaften, wie auch immer sie beschaffen sein mögen, auszuklammern ist. Wohl aber gilt es sich zu fragen, in welchem Maße wir heute einer erneuten fundamentalen Transformation ebenso aller Leseverhältnisse wie auch aller Liebesbeziehungen näher gerückt sind. Dies soll in einer abschließenden Volte unserer Vorlesung prospektiv diskutiert werden! 